LA-UR- $03-2250$

Approved for public release;

distribution is unlimited.
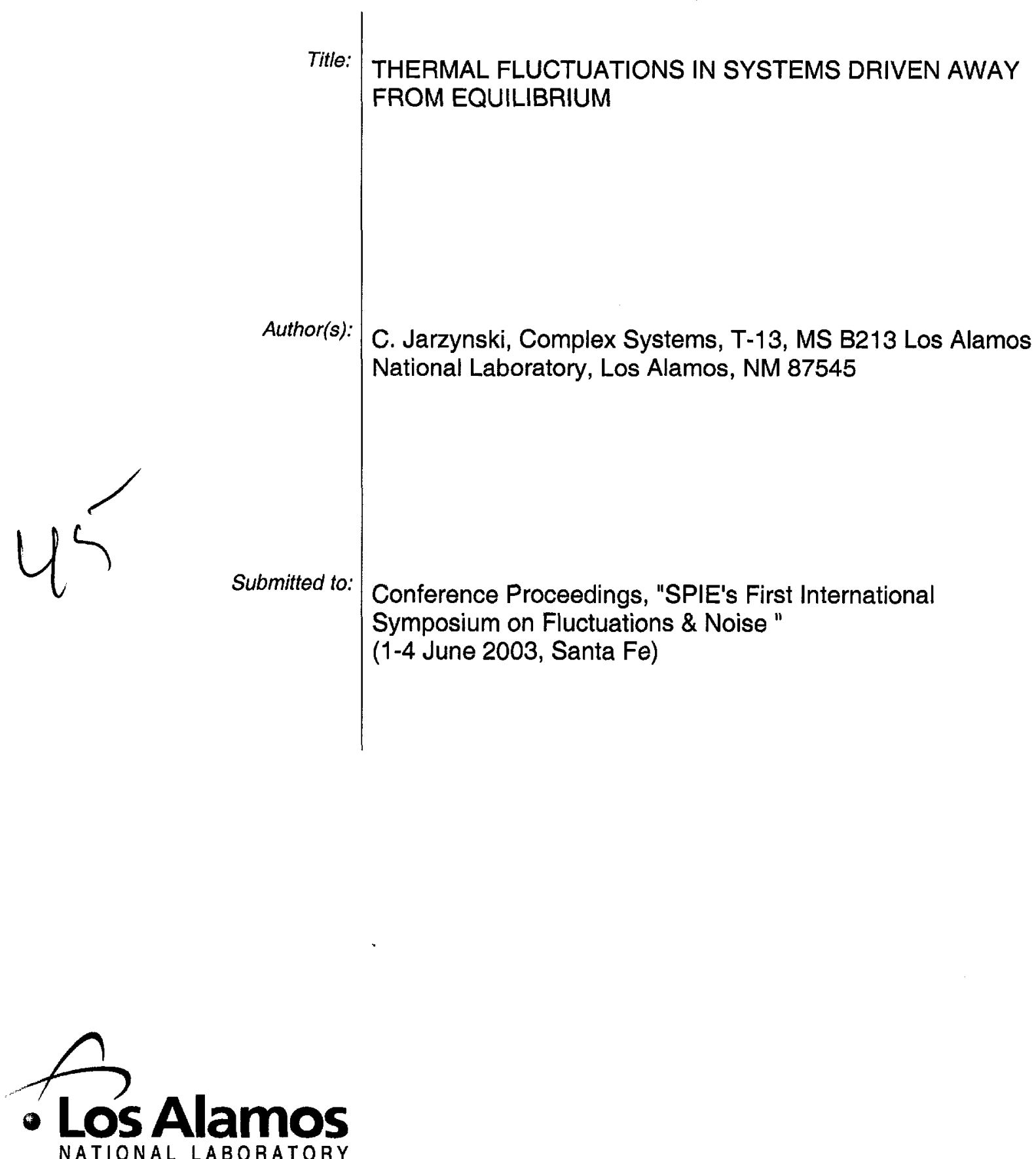

Los Alamos National Laboratory, an affirmative action/equal opportunity employer, is operated $\equiv$ University of California for the U.S. Department of Energy under contract W-7405-ENG-36. By acceptance of this article, the publis = icognizes that the U.S. Government retains a nonexclusive, royalty-free license to publish or reproduce the published form of this collitibution, or to allow others to do so, for U.S. Government purposes. Los Alamos National Laboratory requests that the publisher identify this article as work performed under the auspices of the U.S. Department of Energy. Los Alamos National Laboratory strongly supports academic freedom and a researcher's right to publish; as an institution, however, the Laboratory does not endorse the viewpoint of a publication or guarantee its technical correctness. 


\title{
Thermal fluctuations in systems driven away from equilibrium
}

\author{
C. Jarzynski \\ Complex Systems, T-13, MS B213 \\ Los Alamos National Laboratory \\ Los Alamos, NM 87545 \\ chrisj@lanl.gov
}

\begin{abstract}
I will summarize results that describe the microsopic response of a system driven away from an initial state of thermal equilibrium. I will discuss the application of these results to the analysis of laser tweezer experiments, as well as to the numerical estimation of free energy differences in complex systems such as bio-molecules.
\end{abstract}

\section{INTRODUCTION}

The field of thermodynamics grew out of the study of the efficiency of heat engines, and into a beautiful, logically self-consistent theory that remains unchallenged within its domain of application. Statistical mechanics, in turn, was born of the desire to derive thermodynamic behavior from the underlying motions of atoms and molecules, and to this day much research in statistical mechanics is concerned with systems in the thermodynamic limit of very many degrees of freedom. Nevertheless, from at least the time of Einstein's and Smoluchowski's theories of Brownian motion, it has been understood that the methods of statistical mechanics, properly applied, are useful not only for deriving macroscopic properties from microscopic equations of motion, but also as tools for investigating the behavior of microscopic systems in their own right. 
In this talk I will describe some recent advances in the statistical mechanics of microscopic systems. ${ }^{1-13}$ I will focus on a number of theoretical predictions pertaining specifically to the behavior of systems that are driven away from an initial state of thermal equilibrium, by the external variation of a work parameter. These predictions remain valid even if the system is driven far from equilibrium. This stands in contrast to the much of traditional nonequilibrium statistical mechanics - such as the Einstein relation, Green-Kubo formulae, linear response theory, and so forth - which applies only to systems near thermal equilibrium.

The physical situation mentioned in the previous paragraph - "systems driven away from equilibrium by the variation of a work parameter" - is quite general, and indeed much of the discussion below will take place within the context of a fairly universal set-up. It is always convenient, however, to have in mind an illustrative example to which the theory presented would apply. In the present context single-molecule pulling experiments provide the example. In such experiments the system of interest is a single polymeric molecule, for instance a strand of RNA, immersed in water (typically). By using optical tweezers or atomic force microscopy, one can stretch the molecule and measure the force exerted on it. Such stretching can be carried out slowly, so that the molecule remains in equilibrium with its thermal environment; or it can be carried out rapidly, with the result of driving the molecule out of equilibrium.

Single-molecule pulling experiments can be viewed as the microscopic equivalent of a familiar macroscopic situation, namely the stretching of a rubber band. For this reason, I will begin this talk with a brief summary of the relevant thermodynamics of this macroscopic problem (Section II). This will introduce the roles played by work and free energy in the context of systems driven out of equilibrium. Following that, I will define the set-up for investigating this situation at the microscopic level (Section III). In Section IV, I will present three theoretical predictions applicable to this problem; each of these relates the work $(W)$ performed on the system that is driven out of equilibrium to the free energy difference $(\Delta F)$ between two equilibrium states of the system. Section $V$ places these results in some perspective by relating them to what we already know about reversible processes, 
linear response theory, and the second law of thermodynamics. In Sections VI and VII, I will discuss applications of these theoretical predictions to the analysis of single-molecule stretching experiments, and to the numerical estimation of free energy differences, respectively. A primary aim in these two sections is to illustrate how new and potentially useful results can be obtained by simple algebra, using the results of Section IV.

\section{THERMODYNAMICS OF A STRETCHED RUBBER BAND}

Imagine that I hold taut an ordinary rubber band at a fixed end-to-end distance. I do this under "room conditions": the rubber band is surrounded by air at room temperature and pressure. Thermodynamics states that there is a well defined free energy associated with this equilibrium state of the rubber band. Now I stretch the rubber band, increasing the end-to-end distance. This requires a certain amount of work on my part. If I perform this process very slowly - so that the rubber band is always in equilibrium with the surrounding air - then the amount of work required will equal the difference between the free energies of the initial and final states of the rubber band:

$$
W_{\mathrm{qs}}=\Delta F \equiv F_{B}-F_{A} \text {. }
$$

Here, "qs" stands for quasi-static: the process is carried out very slowly. The subscripts $A$ and $B$ on the right label the initial and final equilibrium states of the rubber band, and $F$ generically denotes free energy.

If I instead stretch the rubber band rapidly, I will notice two things: first, the rubber band heats up, indicating that it is no longer in equilibrium with the surrounding air; second, the tension in the rubber band is greater than in the quasi-static case, which means that I must perform more work:

$$
W>\Delta F
$$

This example contains the basic elements of the physical situation considered in this talk. First, there is a system of interest, the rubber band. This system is in contact with a 
thermal environment, or heat reservoir, i.e. the air surrounding the rubber band. ${ }^{1}$ There is also a work parameter, through which an external agent is able to perform work on the system of interest. In this example, the work parameter is the end-to-end length of the rubber band, which I control externally. Energy can enter or leave the system of interest in the form of heat or work, the former involving energy exchange with the reservoir, the latter involving the work parameter.

The relations between work and free energy differences expressed by Eqs.1 and 2 are not unique to rubber bands: apply universally to thermodynamic processes carried out in the presence of a single heat reservoir. Eq.1 holds if the process is reversible, whereas Eq.2 - a consequence of the second law of thermodynamics - applies to irreversible processes.

The one feature that is missing here, and which will play a central role throughout the rest of the talk, is the notion of fluctuations. Suppose I stretch the rubber band repeatedly, always' starting in the same equilibrium state, and always stretching it, rapidly, in exactly the same manner. Neglecting hysteretic effects, the rubber band will respond in the same way each time I carry out the "experiment". More precisely, any fluctuations that do occur in the response of the rubber band (from one realization of the stretching process to the next) are entirely negligible when compared with the average behavior. However, for genuinely microscopic systems - for instance a single strand of RNA rather than a rubber band fluctuations can be substantial, and therefore of significant interest.

\section{SET-UP}

Let us now move to a microscopic description of the sort of thermodynamic process just illustrated (at the macroscopic level) by the example of the stretched rubber band.

\footnotetext{
${ }^{1}$ Throughout this talk I will assume for simplicity that the system of interest has a fixed number of particles, but the results can be generalized to the situation in which the system and reservoir are in chemical contact, and can exchange particles.
} 
Consider a system of interest whose energy is specified by a Hamiltonian $H(\mathbf{x}, \lambda)$. Here, $\mathbf{x}$ denotes a microstate of the system, i.e. a point in its phase space. The variable $\lambda$ denotes some parameter which we control externally, and through which we perform work on the system. In the context of single-molecule pulling experiments, the system of interest is the chain-like molecule itself, and $\mathbf{x}$ is a many-dimensional vector specifying the position and momentum of each atom constituting the molecule. Typically, one end of the molecule is attached to a microscopic bead, and laser tweezers (for instance) are use to pull on this bead. The work parameter $\lambda$ is then the position of the laser-generated potential used to trap the bead. An alternative set-up is suggested in Ref. ${ }^{9}:$ one end of the molecule is attached to the tip of a microscopic cantilever mounted on a piezo-electrically controlled stage; in this case $\lambda$ is the position of the stage. For specificity we will assume that the stretching is carried out using laser tweezers, but the results discussed below apply equally well to the cantilever set-up.

We will imagine that our system of interest is in contact with a heat reservoir at a temperature $T$. Thus, there are innumerable environmental degrees of freedom with which the system is continually interacting (exchanging energy), with the result that the microstate of the system fluctuates in a seemingly random way. In single-molecule experiments, the environment is of course the water surrounding the molecule. We are not interested in the evolution of the environmental degrees of freedom per se, and so we will not deal with them explicitly, but we will account for their effect on the system of interest.

If $\lambda$ is held fixed, then the system relaxes to a state of thermal equilibrium, in which its fluctuations are characterized by the Boltzmann-Gibbs distribution,

$$
p_{\lambda}^{\mathrm{eq}}(\mathbf{x})=\frac{1}{Z_{\lambda}} e^{-\beta H(\mathbf{x}, \lambda)}
$$

This is the probability distribution of microstates of the system, when it is in equilibrium and the value of $\lambda$ is held fixed; the normalization factor $Z_{\lambda}=\int d \mathbf{x} \exp [-\beta H(\mathbf{x}, \lambda)]$ is the associated partition function; and $\beta=1 / k_{B} T$, as usual. The free energy of this equilibrium state is given by 


$$
F_{\lambda}=-\beta^{-1} \ln Z_{\lambda}
$$

(Strietly speaking, Eq.3 applies only if the interaction energy that couples the system of interest to the environment is negligible. ${ }^{14}$ For an analysis in which non-negligible coupling is explicitly considered, see Ref. ${ }^{15}$.)

Let us now imagine the following thermodynamic process. First, we let the system come to equilibrium with the environment, with the work parameter held fixed at a value $\lambda=A$. This preparation stage takes place prior to time $t=0$. Then, from $t=0$ to $t=\tau$ we externally vary - or "switch" - the work parameter from its initial value $A$ to a final value $B:$

$$
\lambda_{t}=A+(B-A) t / \tau \quad, \quad 0 \leq t \leq \tau
$$

The total switching time, $\tau$, need not be long, i.e. we are free to carry out this process rapidly, if we so choose. As with the rubber band in Section II, the external variation of $\lambda$ has two effects on the system of interest. First, the system is driven out of equilibrium (unless $\lambda$ is varied extremely slowly). Second, we perform work on the system. We will use the variable $w_{t}$ to denote the work performed from time 0 to time $t$, and $W=w_{\tau}$ to denote the total work performed during the switching process. An explicit expression for this work is:

$$
W=\int_{0}^{\tau} d t \dot{\lambda} \frac{\partial H}{\partial \lambda}\left(\mathbf{x}_{t}, \lambda_{t}\right)
$$

where $\dot{\lambda}$ is the rate at which we vary the work parameter. (The expression for $w_{t}$ is the same, except the integral goes from 0 to $t$ rather than 0 to $\tau$.) Note that this work depends both on how we vary the work parameter $\left(\lambda_{t}\right)$ and on how the system evolves in response $\left(\mathbf{x}_{t}\right)$, over the course of the process.

Imagine that we repeat this switching process very many times, always starting in the equilibrium state $A$, and always switching the work parameter from $A$ to $B$ in exactly the same way (Eq.5). Each of these repetitions represents a single realization of the process. Even though the time-dependence of $\lambda$ is always the same, thermal fluctuations guarantee 
that the microscopic evolution of the system of interest will differ from one realization to the next. As a result, the total quantity of work performed will also differ from one realization to the next. It is these fluctuations in the work performed on the system that constitute the focus of this talk. I will use the notation $\rho(W)$ to denote the statistical distribution of work values, i.e. $\rho(W) d W$ is the probability that the work performed will fall between $W$ and $W+d W$, when carrying out this process. Naturally, $\rho(W)$ depends on the initial and final values of the work parameter, $A$ and $B$, as well as on the switching time $\tau$, but in the notation used below this dependence will be implicit rather than explicit.

\section{PREDICTIONS}

In this section I state three theoretical predictions regarding fluctuations in the work performed during the sort of thermodynamic process described in the previous section: Eqs.7, 9, and 12 below. These have by now been derived by a number of authors, using various assumptions regarding the dynamics of the system; I will not present the actual derivations below, but in each case appropriate references to the literature are provided.

The first of these predictions is: ${ }^{1-13,15}$

$$
\left\langle e^{-\beta W}\right\rangle=e^{-\beta \Delta F}
$$

where the angular brackets denote an average over infinitely many realizations of this process, and $\Delta F=F_{B}-F_{A}$ is the free energy difference between the equilibrium states corresponding to the initial and final values of the work parameter $(A$ and $B)$. Explicitly in terms of the distribution of work values, this prediction can be written as:

$$
\int d W \rho(W) e^{-\beta W}=e^{-\beta \Delta F}
$$

Eq.7 relates the work performed during a nonequilibrium (irreversible) process, to a free energy difference between two equilibrium states of the system. Note that we do not assume that the system actually ends in equilibrium. Thus, $F_{B}$ is the free energy associated with 
the final value of the work parameter, rather than the final (possibly out-of-equilibrium) state of the system.

The validity of Eq.7 immediately implies

$$
\left\langle e^{-\beta w_{t}}\right\rangle=e^{-\beta \Delta F_{t}}
$$

where $\Delta F_{t} \equiv F_{\lambda_{t}}-F_{A}$, since we can always define the ending time of our process to be $t$ rather than $\tau$.

The second prediction is slightly more complicated: $:^{2,7,9,15}$

$$
g(\mathbf{x}, t) \equiv\left\langle\delta\left(\mathbf{x}-\mathbf{x}_{t}\right) e^{-\beta w_{t}}\right\rangle=\frac{1}{Z_{A}} e^{-\beta H\left(\mathbf{x}, \lambda_{t}\right)}
$$

Here, the independent variable $\mathbf{x}$ is an arbitrary microstate of the system; $\mathbf{x}_{t}$ is the microstate in which the system is found at time $t$ during a specific realization of the thermodynamic process; $w_{t}$ is (as defined above) the work performed on the system up to time $t$, during this realization; and the angular brackets again denote an average over infinitely many realizations. To gain some intuition for this prediction, note that the function

$$
f(\mathbf{x}, t) \equiv\left\langle\delta\left(\mathbf{x}-\mathbf{x}_{t}\right)\right\rangle
$$

is simply the ordinary ensemble distribution of microstates at time $t$, where the "ensemble" is the set of all possible realizations. By our assumption of initial equilibrium, $f$ is given by the Boltzmann Gibbs distribution at $t=0$, as is $g$ (since $w_{0}=0$ by definition):

$$
f(\mathbf{x}, 0)=g(\mathbf{x}, 0)=\frac{1}{Z_{A}} e^{-\beta H(\mathbf{x}, A)} .
$$

Once the system is driven out of equilibrium $f$ is no longer described by the BoltzmannGibbs formula. Indeed, if the system is driven far from equilibrium by violent changes in the work parameter, then $f$ will evolve in a highly complicated way. Eq.9 tells us, however, that if each realization in the ensemble is assigned a time-dependent statistical weight $\exp \left(-\beta w_{t}\right)$ - which depends on the amount of work performed up to time $t$ during that realization then the evolution of this re-weighted distribution of microstates is very simple: apart from 
normalization, $g$ is the equilibrium distribution corresponding to the current value of the work parameter! As discussed in Ref. ${ }^{2}$, if we picture the ensemble of realizations as a swarm of independent "particles" evolving in (many-dimensional) phase space, and if each particle has a time-dependent "mass" $\exp \left(-\beta w_{t}\right)$, then $f$ can be viewed as a number density, and $g$ as a mass density.

There are two points worth noting immediately about Eq.9. First, by integrating both sides over $\mathbf{x}$, we obtain Eq.8 (or Eq.7 for $t=\tau$ ). Second, at some level Eq.9 represents an answer to the question, How does a system respond when driven away from thermal equilibrium by the external variation of a work parameter? The answer is statistical in nature, and is not necessarily of the form we would like to have: an explicit expression for $f(\mathbf{x}, t)$ would be more useful! Nevertheless, both $f$ and $g$ are valid statistical representations of our ensemble of realizations, and Eq.9 gives us a simple, universally valid expression for the behavior of the latter.

To state the third of the three predictions mentioned above, we will need to consider two different thermodynamic processes. One of these is just the process we have been considering up to this point, in which the system begins in equilibrium state $A$, and then $\lambda$ is varied from $A$ to $B$; we will refer to this as the forward process. The reverse process is then defined as follows: the system begins in equilibrium with the work parameter fixed at $\lambda=B$, then the work parameter is varied from $B$ to $A$ over a time $\tau$, i.e. $\lambda_{t}=B+(A-B) t / \tau$. We imagine that we carry out infinitely many realizations of the forward process, and infinitely many realizations of the reverse process, keeping track of the work performed during each realization. Letting $\rho_{F}(W)$ and $\rho_{R}(W)$ denote the distributions of work values over the two ensembles, the third prediction is: $5,7,11,13,15$

$$
\frac{\rho_{F}(+W)}{\rho_{R}(-W)}=e^{\beta(W-\Delta F)}
$$

where again $\Delta F=F_{B}-F_{A}$. Eq.7 follows immediately from this result by multiplying both sides by $\rho_{R}(-W) \exp (-\beta W)$ and integrating over $W$. There is an amusing consequence of Eq.12: if we plot both $\rho_{F}(+W)$ and $\rho_{R}(-W)$ together, then these two distributions will 


\section{REVERSIBLE PROCESSES, LINEAR RESPONSE, THE SECOND LAW OF THERMODYNAMICS}

We can gain some perspective on Eqs.7, 9 and 12 in the light of other, earlier known results.

The limit $\tau \rightarrow \infty$ is the quasi-static limit, in which the work parameter is switched infinitely slowly from $A$ to $B$ (or $B$ to $A$ for the reverse process). In this case the switching process is reversible, and the work performed is equal to the free energy difference (for every realization), just as in the macroscopic case (Eq.1):

$$
W=W^{\mathrm{qs}}=\Delta F
$$

or

$$
\rho^{\mathrm{qs}}(W)=\delta(W-\Delta F)
$$

(Microscopically, the realizations still differ from one another: if we perform the same quasistatic process repeatedly, then clearly the atoms involved will not be following exactly the same trajectory during each realization. Nevertheless, as a result of quasi-static averaging, the value of $W$ will be the same from one realization to the next.) Eq.7 is clearly satistfied in this case. Indeed, Eq.7 can be viewed as the extension of Eq.13 to irreversible processes.

The work appearing in Eq.13 is the total work performed over the entire process (i.e. up to time $\tau$ ), but the result in fact generalizes to any time between $t=0$ and $t=\tau$ :

$$
w_{t}^{\mathrm{qs}}=F_{\lambda_{t}}-F_{A},
$$

for every realization. This allows us to rewrite the left side of Eq.9 (in the limit $\tau \rightarrow \infty$ ) as:

$$
\exp \left[-\beta\left(F_{\lambda_{t}}-F_{A}\right)\right]\left\langle\delta\left(\mathbf{x}-\mathbf{x}_{\mathbf{t}}\right)\right\rangle
$$

or equivalently 


$$
\frac{Z_{\lambda_{t}}}{Z_{A}} f(\mathbf{x}, t)
$$

But in the quasi-static limit, the system remains in equilibrium at all times, i.e. $f(\mathbf{x}, t)$ is just the Boltzmann distribution associated with the parameter value $\lambda_{t}$. This confirms that Eq.9 is valid in the quasi-static limit.

Let us now move on to nearly-reversible processes, in which the work parameter is varied slowly enough that the system remains very close to equilibrium throughout the process. In this regime, the distribution of work values is not a delta-function (as in Eq.14) but rather a narrow Gaussian:

$$
\rho^{\operatorname{lr}}(W) \propto \exp -\frac{(W-\bar{W})^{2}}{2 \sigma_{W}^{2}}
$$

where "Ir" stands for linear response, $\bar{W}$ is the average work performed on the system, over an ensemble of realizations of the process, and $\sigma_{W}^{2}$ is the variance in these work values. Linear response theory gives an explicit expression for $\sigma_{W}^{2}$ as an autocorrelation function defined with respect to the equilibrium behavior of the system, and - more importantly for present purposes - establishes the following fluctuation-dissipation relation between $\bar{W}$ and $\sigma_{W}^{2}:$

$$
\bar{W}=\Delta F+\frac{1}{2} \beta \sigma_{W}^{2}
$$

With this result it is a straightforward exercise to show that $\rho^{\mathrm{Ir}}$ satisfies Eq.7. Indeed, Eq.7 is equivalent to the following cumulant expansion: ${ }^{1}$

$$
\Delta F=\sum_{n=1}^{\infty}(-\beta)^{n-1} \frac{\omega_{n}}{n !}
$$

where $\omega_{n}$ is the $n^{\prime}$ th cumulant of the distribution of work values (i.e. $\omega_{1}=\bar{W}, \omega_{2}=\sigma_{W}^{2}$, and so forth). Eq.19 is obtained if only the first two terms contribute, which is true if and only if the distribution of work values is a Gaussian.

In the context of Eq.12, if the forward and reverse processes are both nearly reversible, then $\sigma_{W}^{2}$ is the same for both, and $\bar{W}= \pm \Delta F+\frac{1}{2} \beta \sigma_{W}^{2}$, with plus/minus applying in the 
forward/reverse case. By explicitly constructing two Gaussian distributions ( $\rho_{F}$ and $\rho_{R}$ ) with these means and variance, it is straightforward to show that Eq.12 is satisfied.

Having just shown that the Eqs.7, 9 and 12 are consistent with what we know to be true for reversible and nearly reversible processes, it is important to stress that these three predictions remain valid no matter how slowly or quickly the work parameter is varied, even if the system is driven far from equilibrium and linear response no longer holds.

Finally, note that the second law of thermodynamics predicts that $W \geq \Delta F$, with the equality holding only for reversible processes. This law is observed to apply without exception for macroscopic systems, but for microscopic systems it is known that "violations" might occasionally occur. That is, it has long been understood that there exist perfectly valid solutions to Hamilton's equations that are in contradiction with the second law; however, the probability of observing such trajectories spontaneously is expected to become fantastically small with the increasing size of the system under consideration. Eq.7 is consistent with the second law, as can be understood in two ways. First, by Jensen's inequality, an immediate consequence of Eq.7 is that $\langle W\rangle \geq \Delta F$, i.e. that the second law is not violated "on average". Second, following the derivation of Eq.[16] of Ref. ${ }^{6}$, one can show that Eq.7 implies:

$$
\operatorname{Prob}\left[W \leq \Delta F-n k_{B} T\right] \equiv \int_{-\infty}^{\Delta F-n k_{B} T} d W \rho(W) \leq e^{-n}
$$

The left side is the probability that the work performed (during a single realization of the thermodynamic process in question) will fall $n$ or more units of $k_{B} T$ below $\Delta F$; and the right side tells us that this probability is bounded from above by $\exp (-n)$. Here $n$ is an arbitrary positive number. Since any work value less than $\Delta F$ constitutes a "violation" of the second law, this inequality tells us that the probability of violations decreses exponentially (or faster!) in the size of the violation, where size is measure in units of $k_{B} T$. This implies that modest violations $(n \sim 1)$ are not out of the question - and indeed such events have recently been observed experimentally ${ }^{16,17}$ - but macroscopic violations $(n \gg 1)$ are virtually impossible. 


\section{SINGLE-MOLECULE PULLING EXPERIMENTS}

In the context of single-molecule pulling experiments, as mentioned above, the system of interest is the molecule itself, and the work parameter is the position of laser trap used to carry out the stretching. Since the trap acts directly on the microscopic bead to which the molecule is attached, we define our "molecule" to include the bead.

Let us now explicitly separate the energy of the molecule (including attached bead) into an internal part, $H_{0}(\mathbf{x})$, and a biasing potential, $u(z, \lambda)$ :

$$
H(\mathbf{x}, \lambda)=H_{0}(\mathbf{x})+u(z, \lambda) \quad, \quad z=\hat{z}(\mathbf{x}) .
$$

This biasing potential represents the potential energy felt by the system as a result of the laser trap. We assume that this potential acts through a single coordinate, $z$, i.e. the location of the bead along the stretching direction. The notation $\hat{z}(\mathbf{x})$ stresses the explicit functional dependence of this coordinate on the microstate of the molecule.

By direct application of Eq.8, we can estimate the free energy as a function of the work parameter, $\lambda$, from repeated nonequilibrium pulling experiments. Indeed, this has recently been done by Liphardt et $\mathrm{ll}^{16}$, in experiments using a single strand of RNA. These have provided the first direct verification of Eq.7.

Often, it is useful to have the free energy as a function of the pulling coordinate, $z$. Such a free energy profile - in terms of an internal coordinate $z$ rather than an externally controlled parameter $\lambda$ - is a potential of mean force (PMF). Hummer and Szabo ${ }^{9}$ were the first to propose that Eq.9 above could be applied to the analysis of single-molecule pulling experiments, specifically to extract the PMF from a set of nonequilibrium measurements. In what follows I will show how their main result (Eq.31 below) is derived from Eq.9.

The PMF along the coordinate $z$ is defined as:

$$
G_{0}(z) \equiv-\beta^{-1} \ln \frac{\int d \mathbf{x} \delta[z-\hat{z}(\mathbf{x})] e^{-\beta H_{0}(\mathbf{x})}}{\int d \mathbf{x} e^{-\beta H(\mathbf{x}, A)}}
$$

The inclusion of the denominator $Z_{A}$ in this expression is not fundamental $-G_{0}(z)$ is defined only up to an additive constant - but convenient. 
Imagine that we repeatedly carry out the sort of thermodynamic process considered in the previous sections: we start in the equilibrium state corresponding to $\lambda=A$, then we vary the work parameter from $A$ to $B$ over a time $\tau$. During each realization, we note down the evolution of the coordinate $z$ :

$$
z_{t}=\hat{z}\left(\mathbf{x}_{t}\right)
$$

as well as the work performed as a function of time, $w_{t}$.

For an arbitrary function $\phi(z, t)$, consider the quantity

$$
\begin{aligned}
K(z) & \equiv \int_{0}^{\tau} d t\left\langle\delta\left(z-z_{t}\right) e^{-\beta\left[w_{t}-\phi\left(z_{t}, t\right)\right]}\right\rangle \\
& =\int_{0}^{\tau} d t e^{\beta \phi(z, t)} \int d \mathbf{x}\left\langle\delta\left(\mathbf{x}-\mathbf{x}_{t}\right) \delta\left(z-z_{t}\right) e^{-\beta w_{t}}\right\rangle \\
& =\int_{0}^{\tau} d t e^{\beta \phi(z, t)} \int d \mathbf{x} \delta[z-\hat{z}(\mathbf{x})]\left\langle\delta\left(\mathbf{x}-\mathbf{x}_{t}\right) e^{-\beta w_{t}}\right\rangle
\end{aligned}
$$

where the first line defines $K(z)$, and the next two follow by judicious application of basic properties of delta functions. As earlier, angular brackets denote an ensemble average over realizations of the process. Using Eqs.9 and 23 to rewrite the last line, then rearranging terms, we get

$$
K(z)=\int_{0}^{\tau} d t \exp \beta\left[\phi(z, t)-u\left(z, \lambda_{t}\right)-G_{0}(z)\right],
$$

from which we finally derive the following general expression for the PMF:

$$
G_{0}(z)=-\beta^{-1} \ln \frac{\int_{0}^{\tau} d t\left\langle\delta\left(z-z_{t}\right) \exp -\beta\left[w_{t}-\phi\left(z_{t}, t\right)\right]\right\rangle}{\int_{0}^{\tau} d t \exp -\beta\left[u\left(z, \lambda_{t}\right)-\phi(z, t)\right]} .
$$

We are free to choose the function $\phi(z, t)$ appearing in Eq.29, as the derivation of this result does not depend on any assumptions regarding $\phi$. In a practical application, of course, the average appearing in Eq.29 is estimated with the data from a finite number of repetitions of the pulling experiment, and the amount of error due to finite sampling will depend very much on the choice of $\phi$. A choice that naturally comes to mind is $\phi(z, t)=u\left(z, \lambda_{t}\right)$, which gives: ${ }^{2}$

${ }^{2}$ This result also follows immediately from Eq.[7] of Ref. ${ }^{9}$. 


$$
G_{0}(z)=-\beta^{-1} \ln \left[\frac{1}{\tau} \int_{0}^{\tau} d t\left\langle\delta\left(z-z_{t}\right) e^{-\beta \Delta w_{t}}\right\rangle\right],
$$

using the notation $\Delta w_{t} \equiv w_{t}-u\left(z_{t}, \lambda_{t}\right)$. The expression for the PMF derived by Hummer and Szabo corresponds to the choice $\phi(z, t)=F_{\lambda_{t}}-F_{A} \equiv \Delta F_{t}$ :

$$
G_{0}(z)=-\beta^{-1} \ln \frac{\int_{0}^{\tau} d t\left\langle\delta\left(z-z_{t}\right) \exp -\beta\left(w_{t}-\Delta F_{t}\right)\right\rangle}{\int_{0}^{\tau} d t \exp -\beta\left[u(z, t)-\Delta F_{t}\right]} .
$$

In practice, $\Delta F_{t}$ is not known; rather, our estimate of its value is refined through an iterative process until the solution of $G_{0}(z)$ is consistent with the estimate of $\Delta F_{t}$ appearing on the right. ${ }^{9}$

\section{FREE ENERGY CALCULATIONS}

The problem of obtaining accurate and efficient numerical estimates of equilibrium free energy differences is both challenging and of considerable practical importance..$^{18-20}$ One class of methods developed for this problem involves the numerical simulation of the sort of thermodynamic process considered throughout this talk.

Suppose we have two Hamiltonians $H_{0}(\mathbf{x})$ and $H_{1}(\mathbf{x})$ defined on the same phase space, and we want to compute the free energy difference between the corresponding canonical ensembles, at temperature $T$. We can proceed by defining a parameter-dependent family $H(\mathbf{x}, \lambda)$, such that $H_{0}$ and $H_{1}$ correspond to two values of the parameter, e.g.

$$
H_{0}(\mathbf{x})=H(\mathbf{x}, A) \quad, \quad H_{1}(\mathbf{x})=H(\mathbf{x}, B) .
$$

We can then run a long numerical simulation in which the system begins in the equilibrium state $A$, and then evolves in time as the work parameter is switched slowly from $A$ to $B$. We keep track of the work performed on the system during this in silico switching process. If the simulation is carried out sufficiently slowly, then the total work performed provides a good estimate of the free energy difference $\Delta F$, by Eq.13.

Alternatively, we can divide our available computer time (or processors) among a number of simulations carried out independently of one another. Viewing these as different realizations of the same thermodynamic process, we can then apply Eq.7 to construct an estimate 
of $\Delta F$. This estimate does not rely on the assumption that each realization is performed nearly quasi-statically: in principle the simulations can be carried out rapidly.

These methods are sometimes known as slow growth (one long simulation) and fast growth (many shorter simulations). While fast growth might have some advantages over slow growth ${ }^{21}$, it is by no means a silver bullet. Fast growth indeed allows us to drop the computationally burdensome requirement of simulating quasi-static processes, but there is a price to pay: the shorter the duration of each simulation, the more of them we need in order for the average of $\exp (-\beta W)$ to converge to a reasonable estimate. There is thus considerable room for improvement over the straightforward application of Eq.7, and indeed a number of strategies have already been suggested. For a sample of recent publications related to the estimation of free energy differences using nonequilibrium simulations, see Refs. ${ }^{22-28}$.

In the remainder of this section I will show how an alternative method for estimating $\Delta F$ can be constructed from Eq.12. I will assume that simulations are performed in both the forward $(F)$ and the reverse $(R)$ directions, i.e. from $\lambda=A$ to $\lambda=B$, and vice-versa. The work values emerging from these simulations can be viewed as independent values of $W$ sampled from the distributions $\rho_{F}(W)$ and $\rho_{R}(W)$. The method obtained below is essentially a generalization of the acceptance ratio method derived by Bennett ${ }^{11,29}$. In its original form the acceptance ratio method applies to values of the phase space function $\Delta H \equiv H_{1}-H_{0}$ sampled from the equilibrium (canonical) ensembles associated with the Hamiltonians $H_{0}$ and $H_{1}$. Eq.35 below extends this method to values of work performed during forward and reverse nonequilibrium switching simulations. ${ }^{7}$

Letting $C$ denote an adjustable parameter that has units of energy, we can multiply both sides of Eq.12 by $\exp (\beta C)$, then rearrange terms to obtain

$$
\rho_{F}(W) e^{\beta(C-W)}=e^{\beta(C-\Delta F)} \rho_{R}(-W) .
$$

Next, letting $f$ denote the Fermi-Dirac function, $f(x) \equiv 1 /[1+\exp (\beta x)]$, then multiplying both sides by $f(C-W)$ and integrating over $W$, we get 


$$
\langle f(W-C)\rangle_{F}=e^{\beta(C-\Delta F)}\langle f(W+C)\rangle_{R},
$$

where the subscripts $F$ and $R$ denote averages over work values generated during the forward and reverse simulations, respectively. This finally leads to

$$
\beta \Delta F=\ln \frac{\langle f(W+C)\rangle_{R}}{\langle f(W-C)\rangle_{F}}+\beta C .
$$

Eq.35 is formally valid for any value of $C$. In practice, we estimate the two averages appearing in this equation by running a finite number of forward and reverse simulations. These estimates will therefore be subject to statistical errors, whose size can depend strongly on $C$. It can be shown ${ }^{11,29}$ that the optimal value of $C$ - the one that minimizes the expected statistical error - is given by

$$
C=\Delta F .
$$

This is of course precisely the quantity that we are trying to estimate! We therefore construct a best estimate of $\Delta F$ by demanding consistency between Eqs.35 and 36: we adjust $C$ until both equations are satisfied. This procedure can be understood graphically. Eq.35 implies that the functions $\eta_{R}(C) \equiv\langle f(W+C)\rangle_{R}$ and $\eta_{F}(C) \equiv\langle f(W-C)\rangle_{F}$ intersect precisely at $C=\Delta F$. Demanding consistency between Eqs.35 and 36 thus amounts to searching for the point of interesection between $\eta_{R}$ and $\eta_{F}$.

In his 1976 paper Bennett also introduced the overlapping distributions method ${ }^{11,29}$, which makes use of $\Delta H$ values sampled from equilibrium distributions (as with Bennett's version of the acceptance ratio method). It has been suggested by Frenkel ${ }^{30}$ that the overlapping distributions method can be generalized, so that it applies also to work values performed during nonequilibrium switching simulations. That this is indeed the case is easy to establish, again taking Eq.12 as the starting point.

\section{CONCLUSIONS}

In this talk I have attempted - without going into the details of formal derivations to summarize a number of salient features of recent theoretical predictions pertaining to 
systems driven away from equilibrium. Perhaps the most important of these is the fact that these predictions are not restricted to the near-equilibrium regime, but remain valid even far from equilibrium.

From the perspective of fundamental statistical mechanics, the results discussed in Section IV help us to understand the nature of the second law of thermodynamics as it applies to microscopic systems. In this respect these results have much in common with the fluctuation theorem ${ }^{12,17,31}$, which has been derived for systems evolving in (or toward) nonequilibrium steady states. Indeed, Crooks ${ }^{5}$ has established the close relationship between these two sets of far-from-equilibrium predictions.

From a practical standpoint, the experiments of Liphardt et al ${ }^{16}$ provide not only a validation of Eq.7, but also optimism that these predictions will be of actual use in the analysis of experimental data, for instance as proposed by Hummer and Szabo. ${ }^{9}$

Finally, Eqs.7, 9, and 12 have emerged as a promising tool in the numerical estimation of free energy differences. In this area in particular it seems that much work remains to be done. At the very least, however, we now know that it is possible to extract equilibrium information from simulations of nonequilibrium processes.

This work is supported by the U.S. Department of Energy, under contract W-7405-ENG36. 


\section{REFERENCES}

${ }^{1}$ C.Jarzynski, Phys.Rev.Lett.78, 2690 (1997).

${ }^{2}$ C.Jarzynski, Phys.Rev.E 56, 5018 (1997).

${ }^{3}$ G.E.Crooks, J.Stat.Phys.90, 1481 (1998).

${ }^{4}$ C.Jarzynski, Acta Phys.Polonica B 29, 1609 (1998).

${ }^{5}$ G.E.Crooks, Phys.Rev.E 60, 2721 (1999).

${ }^{6}$ C.Jarzynski, J.Stat.Phys. 96, 415 (1999).

${ }^{7}$ G.E.Crooks, Phys.Rev.E 61, 2361 (2000).

${ }^{8}$ S.Yukawa, J.Phys.Soc.Japan 69, 2367 (2000).

${ }^{9}$ G.Hummer and A.Szabo, Proc.Natl.Acad.Sci.(USA) 98, 3658 (2001).

${ }^{10}$ R.M.Neal, Statistics and Computing 11, 125 (2001).

${ }^{11}$ D.Frenkel and B.Smit, Understanding Molecular Simulation: From Algorithms to Applications, second edition, Chapter 7. Academic Press, San Diego (2002).

${ }^{12}$ C. Maes and K. Netočný, J. Stat. Phys. 110, 269 (2003).

${ }^{13}$ D.J.Evans, "A nonequilibrium free energy theorem for deterministic systems", submitted for publication.

${ }^{14}$ See, e.g. F. Reif, Fundamentals of Statistical and Thermal Physics (McGraw-Hill, New York, 1965), pp. 95, 202.

${ }^{15}$ C.Jarzynski, "Far-from-equilibrium response in the presence of strong coupling to environment", manuscript in preparation.

${ }^{16}$ J.Liphardt, S.Dumont, S.B.Smith, I.Tinoco, C.Bustamante, Science 296, 1832 (2002).

${ }^{17}$ G.M.Wang, E.M.Sevick, E.Mittag, D.J.Searles, D.J.Evans, Phys.Rev.Lett.89, 050601 
(2002).

${ }^{18}$ See, for instance, M.R.Reddy and M.D.Erion, eds, Free Energy Calculations in Rational Drug Design, Kluwer Publishing, 2001.

${ }^{19}$ C.Chipot and D.A.Pearlman, Mol.Simul. 28, 1 (2002).

${ }^{20}$ A.H.Elcock, Curr.Opin.Struct.Biol. 12, 154 (2002).

${ }^{21}$ D.A.Hendrix and C.Jarzynski, J.Chem.Phys.114, 5974 (2001).

${ }^{22}$ G.Hummer, J.Chem.Phys. 114, 7330 (2001).

${ }^{23}$ H.Nanda, D.M.Zuckerman, T.B.Woolf, Biophys.J. 82, 328A (2002).

${ }^{24}$ D.M.Zuckerman and T.B.Woolf, Chem.Phys.Lett. 351, 445 (2002).

${ }^{25}$ M.O.Jensen, S.Park, E.Tajkhorshid, K.Schulten, Proc.Natl.Acad.Sci.(USA) 99, 6731 (2002).

${ }^{26}$ H.Hu, R.H.Yun, J.Hermans, Mol.Simul. 28, 67 (2002).

${ }^{27}$ M.Athenes, Phys.Rev.E 66, 046705 (2002).

${ }^{28}$ D.M.Zuckerman and T.B.Woolf, Phys.Rev.Lett.89, 180602 (2002).

${ }^{29}$ C.H.Bennett, J.Comp.Phys.22, 245 (1976).

${ }^{30}$ Daan Frenkel, personal communication.

${ }^{31}$ See for instance D.J.Evans and D.J.Searles, Adv.Phys.51, 1529 (2002), and many references therein. 